\title{
Propiedades mecánicas y tribológicas de materiales nanoestructurados de carburo de silicio/nanofibras de carbono
}

\author{
A. BORRELL', R. TORRECILLAS', V.G ROCHA², A. FERNÁNDEZ², V. BONACHE³, M.D. SALVADOR \\ ${ }^{1}$ Centro de Investigación en Nanomateriales y Nanotecnología (CINN) (Consejo Superior de Investigaciones Científicas - Universidad de Oviedo - \\ Principado de Asturias), Parque Tecnológico de Asturias, 33428 Llanera (Asturias), Spain \\ 2ITMA Materials Technology, Parque Tecnológico de Asturias, 33428 Llanera (Asturias), Spain \\ ${ }^{3}$ Instituto de Tecnología de Materiales (ITM), Universidad Politécnica de Valencia, Camino de Vera, s/n, 46022 Valencia, Spain
}

\begin{abstract}
Los materiales compuestos cerámica/ carbono poseen excelentes propiedades que los convierten en candidatos idóneos para diversas aplicaciones. Entre otras cabe destacar su empleo en frenos de aviones o coches de competición. Su baja densidad, alta resistencia mecánica, elevada resistencia a la oxidación, excelente comportamiento a fricción y una baja sensibilidad a las condiciones de operación permite utilizar estos compuestos como material funcional. Por todo ello, la obtención de nuevos materiales nanoestructurados cerámica/carbono aparece como una opción muy interesante dada la creciente demanda que existe desde las industrias automovilística y aeronáutica. El objetivo de este trabajo es evaluar la influencia de las nanofibras de carbono (CNFs) en el comportamiento mecánico y tribológico de los nanocompuestos de SiC/CNFs obtenidos mediante la técnica de spark plasma sintering. La caracterización tribológica realizada se ha basado en ensayos de deslizamiento en seco, mediante un dispositivo tipo ball-on-disk. La carga de contacto que se ha utilizado ha sido de $15 \mathrm{~N}$. Las superficies de desgaste se han observado mediante microscopía electrónica de barrido. Los resultados obtenidos demuestran la mejora simultánea del comportamiento al desgaste y de las prestaciones mecánicas del material cerámico al incorporarle las nanofibras de carbono, siendo el coeficiente de fricción muy estable.
\end{abstract}

Palabras clave: Compuestos nanofibras de carbono/carburo de silicio, Spark plasma sintering, Propiedades mecánicas, Tribología, Desgaste.

\section{Mechanical properties and tribological behaviour of silicon carbide/carbon nanofibers nanocomposites}

The development of new ceramic/carbon nanostructured materials is a very interesting option from the point of view of the automotive and aerospace industries. Its low density, high mechanical strength, high oxidation resistance and excellent friction behavior allows the use of these composites as functional materials. The aim of this study was to evaluate the influence of carbon nanofibers (CNFs) on the mechanical and tribological behavior of silicon carbide/CNFs nanocomposite obtained by spark plasma sintering technique. The tribological study was carried out in a ball-on-disk apparatus under dry sliding conditions (dry friction) and a fixed load of $15 \mathrm{~N}$. The friction coefficient and wear rate were measured for each composite. Scanning electron microscope was used to analyze wear surface formed. The results show simultaneous improvement of wear behavior and mechanical properties of ceramic materials by incorporating of carbon nanofibers.

Keywords: Carbon nanofibers/silicon carbide nanocomposites, Spark plasma sintering, Mechanical properties, Tribology, Wear.

\section{INTRODUCCIÓN}

El estudio de las propiedades tribológicas y mecánicas de los materiales es de gran importancia para el éxito en el diseño de piezas y mecanismos de trabajo, a fin de evitar que los esfuerzos a que se encuentran sometidas causen deformaciones, fisuras y desgaste superficial de los componentes. Los materiales compuestos cerámica/carbono poseen excelentes propiedades para diversas aplicaciones: materiales refractarios, materiales estructurales para uso a temperaturas intermedias, materiales resistentes a la corrosión y materiales como revestimientos de frenos, entre otras $(1,2)$. Estos materiales compuestos de carbono se caracterizan por su alta resistencia (incluso a elevadas temperaturas), su gran tenacidad, bajo coeficiente de expansión térmica y buena conductividad eléctrica. Además, el mantenimiento de sus propiedades a elevadas temperaturas, hace de los materiales compuestos cerámica/carbono la única solución potencial para un gran número de aplicaciones en el campo de la ingeniería $(3,4)$. Por lo tanto, la obtención de nuevos materiales compuestos cerámica/carbono, mediante una tecnología que permita reducir los costes de fabricación, aparece como una opción muy interesante, dada la enorme demanda que existe desde las industrias automovilística y aeronáutica. Esta reducción en los costes de fabricación debe ir acompañada de la conservación, e incluso mejora, de las propiedades finales del material. Una posible solución la constituyen los materiales nanocompuestos. En los últimos años, han sido numerosos los ejemplos en los que la obtención de materiales nanoestructurados ha supuesto una enorme 
mejora en las propiedades finales en comparación con los equivalentes micrométricos (5). Los materiales cerámicos de mayor interés tribológico son los que combinan baja densidad con excelentes propiedades mecánicas $\left(\mathrm{Si}_{3} \mathrm{~N}_{4}, \mathrm{SiC} \mathrm{o} \mathrm{Al} \mathrm{O}_{3}\right)$. El carburo de silicio ( $\mathrm{SiC}$ ) es el material cerámico no oxídico más empleado en la industria desde hace más de 100 años. Debido a sus excelentes propiedades tanto eléctricas como mecánicas a altas temperaturas, es considerado una importante cerámica estructural (6). Las nanofibras de carbono, debido a su tamaño nanométrico, a su estructura altamente grafítica, a sus características morfológicas y a sus excepcionales propiedades mecánicas y de transporte eléctrico y térmico, tienen un excelente potencial como material ingenieril (7).

Hasta el momento, la investigación sobre la obtención de nanocompuestos densos y homogéneos de carburo de silicio y nanofibras de carbono no es muy extensa, tal vez porque sigue siendo un desafío técnico. Shimoda et al. (8) estudiaron la preparación de nanocompuestos de $\mathrm{SiC} / \mathrm{CNF}$ con un 1-10 $\%$ en peso de CNFs por prensado en caliente (hot-press) a $1900{ }^{\circ} \mathrm{C}$ durante $1 \mathrm{~h}$, utilizando como aditivos de sinterización partículas sub-micrométricas de $\mathrm{Al}_{2} \mathrm{O}_{3}$ y $\mathrm{Y}_{2} \mathrm{O}_{3}$. Los autores encontraron que los composites $\mathrm{SiC} / \mathrm{CNF}$ con un contenido de CNFs por debajo del $5 \%$ en peso, alcanzaron densidades del $98 \%$ con una buena dispersión de las CNFs. Sin embargo, los nanocomposites $\mathrm{SiC} / \mathrm{CNF}$ con un contenido de un $10 \%$ en peso de CNFs mostraban densidades relativas del 92\% con presencia de aglomerados de CNFs. Por otro lado, aunque en el trabajo de Hirora et al. (9) se estudia la síntesis y consolidación simultanea mediante la técnica de Spark Plasma Sintering (SPS) de los composites SiC/CNFs, en dicho trabajo se utilizan boro y carbono amorfo como aditivos. Se obtuvieron composites $\mathrm{SiC} / 10 \%$ vol. CNFs densos ( 96\% de la densidad teórica) a $1800{ }^{\circ} \mathrm{C}$ durante $10 \mathrm{~min}$, con unas propiedades mecánicas mejoradas $\sim 30 \%$ en comparación con el material de $\mathrm{SiC}$ monolítico.

En el presente trabajo se estudia el comportamiento tanto mecánico como tribológico de los materiales compuestos obtenidos a partir de nanofibras de carbono y carburo de silicio. Estos nanocompuestos han sido sinterizados sin añadir ningún tipo de aditivo específico a diferentes temperaturas finales y tiempos de mantenimiento, utilizando la técnica de sinterización rápida basada en descargas eléctricas, SPS (del inglés, Spark Plasma Sintering) (10-12).

\section{PROCEDIMIENTO EXPERIMENTAL}

Las nanofibras de carbono (CNFs) utilizadas en este trabajo han sido suministradas por el Grupo Antolín Ingeniería (Burgos, España), presentan un diámetro entre 20-80 nm y una longitud de hasta 30 micras, siendo la mayoría de ellas mucho más pequeñas con longitudes comprendidas entre 5-10 micras. Estas CNFs han sido generadas en fase vapor (VGCNFs) (13), utilizando un catalizador flotante de níquel en disolución (6-8\%). Los polvos de nano-carburo de silicio $(\beta-\mathrm{SiC})$ comerciales que se han utilizado fueron suministrados por Hubei Corporation (China), cuyo tamaño de partícula promedio es de $50 \mathrm{~nm}$ y tienen una pureza $>98 \%$, la estructura cristalina es la cúbica 3C-SiC. Se le ha realizado una difracción de rayos $X$ a los polvos de $\beta$-SiC de partida y se ha comprobado que la posición e intensidad de los picos observados se ajustan a la ficha ICDD (Base de datos del "International Centre for Diffraction Data") número 29-1129. La preparación de las mezclas de polvos se llevó a cabo utilizando una sonda ultrasónica de alta potencia (Dr. Hielscher, Germany), aplicando una frecuencia de $70 \mathrm{~A}$ durante 15 minutos. Posteriormente, la suspensión se secó a 60 ${ }^{\circ} \mathrm{C}$ y a $120^{\circ} \mathrm{C}$. El polvo resultante se tamizó a $<63$ micras. Las composiciones que se estudiaron fueron, $\mathrm{nSiC} / \mathrm{CNFs}=100 / 0$ - 80/20 - 50/50 - 20/80 - 0/100 \%vol. Las mezclas de polvos obtenidas fueron sinterizadas en un equipo SPS modelo HP D 25/1, FCT (Systeme GmbH, Rauenstein, Germany) a las temperaturas finales de 1600 y $1800{ }^{\circ} \mathrm{C}$, aplicando una presión uniaxial de $80 \mathrm{MPa}$ y distintos tiempos de estancia a la máxima temperatura, 1 y 30 minutos. En cada ensayo se utilizaron de 2.2 a 3.2 g de material, dependiendo de la mezcla, que se introdujeron en un molde de grafito de 20 $\mathrm{mm}$ de diámetro. La velocidad de calentamiento utilizada fue de $100{ }^{\circ} \mathrm{C} / \mathrm{min}$ en vacío. Se sinterizaron al menos cinco probetas de cada mezcla preparada. La densidad geométrica de cada material se determinó a partir del peso y del volumen geométrico del material, siendo la pieza un disco regular de $20 \mathrm{~mm}$ de diámetro. Los espectros Raman se obtuvieron en un espectrómetro Raman modular XY, de la casa DILOR, con un detector CCD4. La fuente de excitación utilizada fue un láser de Árgon, modelo Specra-Physics 265, del que se utilizó la raya verde de longitud de onda de $532 \mathrm{~nm}$. La potencia de láser utilizada fue de $20 \mathrm{~mW}$ y la anchura de la rendija de $200 \mu \mathrm{m}$. Los espectros obtenidos fueron deconvolucionados para separar los picos mediante un ajuste a curvas de tipo lorentziano/gaussiano y llevar a cabo el cálculo de los parámetros. La posición y anchura de las bandas se utilizaron para estimar el grado de orden de los materiales. Para medir la resistencia a la fractura de los diferentes materiales obtenidos se utilizó el ensayo biaxial de acuerdo con la ecuación de Kirstein y Woolley (14) y Vitman y Pukh (15), mediante la especificación estándar ASTM F394-78 (16). Los ensayos fueron realizados a temperatura ambiente usando la máquina universal (Instron Model 8562), con una velocidad de desplazamiento de carga de 0.002 $\mathrm{mm} / \mathrm{s}$. Los ensayos de desgaste han sido realizados con deslizamiento en seco, utilizando un dispositivo experimental del tipo "ball-on-disk". En este ensayo el material a ensayar actúa de plato y como contramaterial se ha empleado una

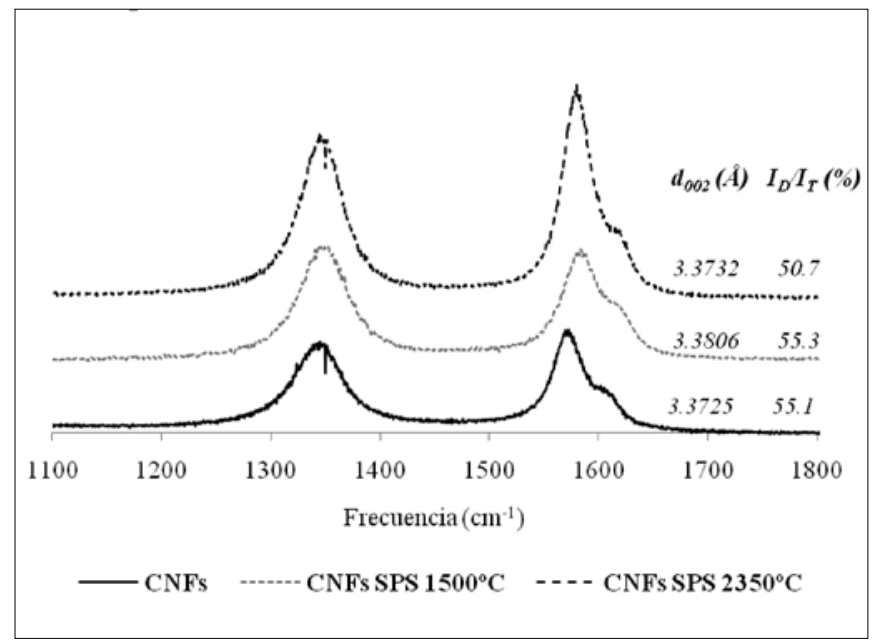

Figura 1. Espectro Raman de primer orden de las CNFs de partida y de las CNFs tras el tratamiento en el SPS a distintas temperaturas. 
bola cerámica de alúmina de $5 \mathrm{~mm}$ de diámetro. El sistema dispone de un par de células de carga que permiten medir el coeficiente de rozamiento en cada momento. El desgaste del material es determinado mediante la diferencia de peso usando una microbalanza de $10 \mu \mathrm{g}$ de precisión. Los ensayos fueron realizados a temperatura ambiente con una humedad controlada del $60 \%$, la carga de contacto utilizada fue de $15 \mathrm{~N}$, la velocidad de $250 \mathrm{rpm}$ y una distancia de deslizamiento de $1000 \mathrm{~m}$. Estos estudios fueron completados con la observación de las huellas de desgaste mediante microscopía electrónica de barrido (MEB, Zeiss DSM 950), con el fin de analizar los mecanismos de daño involucrados.

\section{RESULTADOS}

\subsection{Efecto de la temperatura final de sinterización: Materiales sinterizados a $1600{ }^{\circ} \mathrm{C}$ y $1800{ }^{\circ} \mathrm{C}$.}

En el caso de los materiales cerámicos compuestos que incorporan nanoestructuras de carbono, los cuales necesitan elevada temperatura para poder densificarlos, la integridad de esas nanoestructuras puede verse comprometida, por lo se ha realizado previamente la caracterización mediante micro-Raman de los materiales antes y después del proceso de sinterización. En la Figura 1 se representan los espectros Raman de las CNFs de partida y de dos materiales sinterizados a $1500{ }^{\circ} \mathrm{C}$ y $2350{ }^{\circ} \mathrm{C}$. La selección de dichas temperaturas tiene como objetivo evaluar la transformación sufrida por las CNFs a una temperatura similar a las comúnmente utilizadas en el procesamiento de nanocomposites CNFs-cerámica (1400$\left.1800{ }^{\circ} \mathrm{C}\right) \mathrm{y}$, por otro lado, someter a las CNFs de carbono a unas condiciones de sinterización extremas, como sería 2350 ${ }^{\circ} \mathrm{C}$. Como se puede observar, no se aprecia una modificación importante, ni en el espectro ni en los parámetros calculados a partir del mismo, en el caso del material sinterizado a $1500^{\circ} \mathrm{C}$. Tal y como cabría esperar, hay una reducción en la intensidad relativa de la banda $\mathrm{D}$, correspondiente al desorden, cuando el material es sinterizado a temperaturas superiores a 2000 ${ }^{\circ} \mathrm{C}$. Con el fin de facilitar la observación de la evolución de las bandas del espectro Ramán, en la Figura 1 se han incluido los resultados correspondientes a materiales obtenidos a partir de

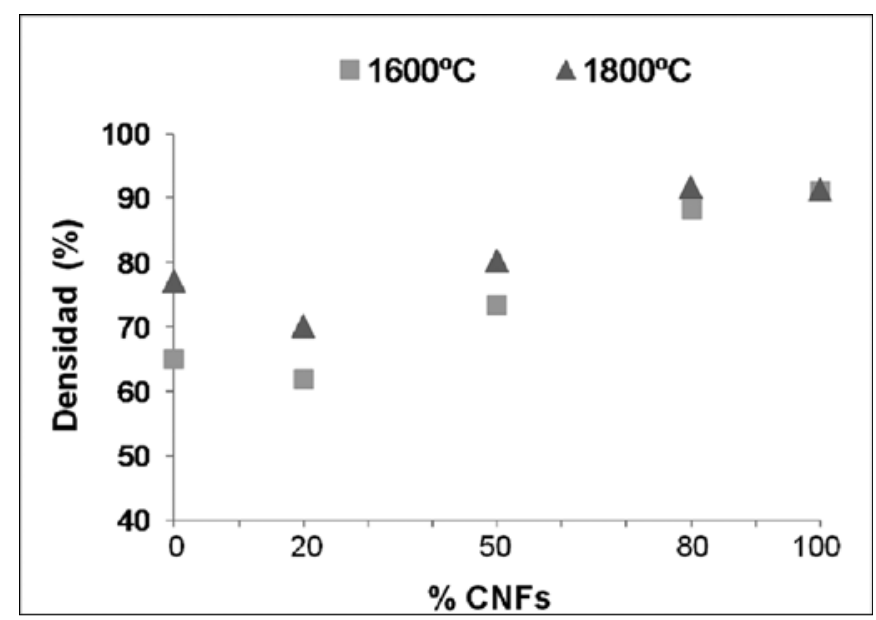

Figura 2. Densidad relativa de los composites de nSiC/CNFs sinterizados a distintas temperaturas con 1 minuto de estancia a la máxima temperatura.
$100 \%$ CNFs. Los materiales compuestos SiC-CNFs han sido sinterizados a 1600 y $1800{ }^{\circ} \mathrm{C}$ durante 1 min de estancia a la máxima temperatura. La evolución de la intensidad relativa de la banda D es similar, con valores alrededor de $55-53 \%$.

Se han determinado sus densidades, cuyos valores se representan en la Figura 2 en función del contenido en nanofibras de carbono. El error experimental calculado asociado al valor medio fue de $\pm 0.5 \%$. Los resultados mostrados en dicha figura indican que tiene lugar un aumento de densidad en el material final al aumentar el contenido en nanofibras de carbono, consiguiéndose densidades $>90 \%$ con contenidos del $80 \%$ vol. de CNFs. Por otro lado, a medida que el composite tiene un mayor contenido en carburo de silicio, el valor de la densidad alcanzada es más sensible a la temperatura final de sinterización.

La densificación de los composites de nSiC/CNFs es especialmente complicada ya que además de ser necesaria una buena dispersión de las nanofibras de carbono con el carburo de silicio, el proceso de sinterización y los mecanismos de densificación de los materiales con fuertes enlaces covalentes, como es el caso del carburo de silicio, presenta una elevada dificultad a la hora de obtener materiales cerámicos densos. Estos enlaces, al contrario que los iónicos o los metálicos, presentan una baja difusividad atómica y una alta energía en la frontera de grano, haciendo que el material tenga una alta temperatura de descomposición $\left(2545^{\circ} \mathrm{C}\right)$, al mismo tiempo que dificulta su sinterización (17). Por lo tanto, el uso de pequeñas cantidades de aditivos como el boro y el carbono han sido utilizados tradicionalmente para la sinterización del SiC, tanto en horno convencional (18) como en Hot-Press (19), obteniéndose de esta forma materiales cerámicos densos. Estos "activadores de sinterización" se utilizan en cantidades de $0.5-3 \%$ en peso, siendo estas las cantidades óptimas para obtener unas buenas propiedades mecánicas incluso a alta temperatura (20). El papel que juegan los aditivos en el proceso de sinterización es todavía una cuestión abierta y se cree que la función que tiene cada uno de los elementos es diferente. En general, se considera que el boro contribuye a la movilidad de los átomos dentro de los límites de la frontera de grano, ayudando a que se produzca un aumento en el número de

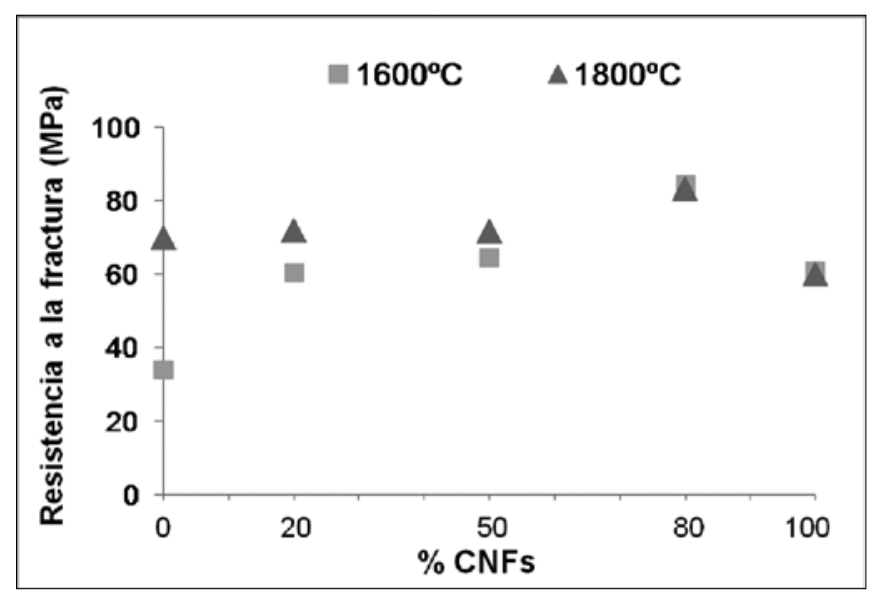

Figura 3. Resistencia a la fractura de los composites nSiC/CNFs sinterizados a distintas temperaturas con 1 minuto de estancia a la máxima temperatura. 
contactos de grano y, por lo tanto, una disminución en la porosidad de mayor tamaño que no puede ser eliminada durante la sinterización. Por otro lado, el carbono ayuda a inhibir el crecimiento de grano haciendo que la porosidad que se forma entre los bordes de grano sea menos significativa (20).

Los composites $\mathrm{nSiC} / \mathrm{CNFs}$ estudiados en este trabajo a pesar de haber sido sinterizados sin añadir ningún tipo de aditivo específico, ya que uno de los objetivos del presente trabajo es estudiar la influencia que tienen las nanofibras de carbono en la densificación del carburo de silicio, presentan densidades significativamente altas a medida que el porcentaje en CNFs es más alto. Esta tendencia es totalmente opuesta a lo que ocurre en los composites $\mathrm{Al}_{2} \mathrm{O}_{3} / \mathrm{CNFs}$ y $\mathrm{ZrO}_{2} / \mathrm{CNF}$, estudiados previamente por los autores (21-23), lo que parece indicar que las propias nanofibras de carbono actúan en cierta medida como aditivo de sinterización, mejorando de esta forma la densidad de los composites con $\mathrm{nSiC}$.

La caracterización mecánica de los composites de carburo de silicio con nanofibras de carbono se ha enfocado al estudio de la variación de la resistencia a la fractura de los materiales, con su composición y la temperatura de sinterización utilizada. Estos resultados se muestran en la Figura 3. El error experimental calculado asociado al valor medio fue de \pm 5 $\mathrm{MPa}$. Se observa que los valores de resistencia a la fractura de los composites aumentan a medida que los porcentajes de nanofibras de carbono son más altos y la temperatura se incrementa, lo que se puede relacionar directamente con la densidad de los materiales, como se observó anteriormente. Más allá de este comportamiento, un resultado interesante es que, al contrario de lo que ocurre con la variación de la densidad que sigue una tendencia, aproximadamente, lineal con el aumento del contenido en nanofibras de carbono, en este caso, la resistencia a la fractura de todos los materiales nanocompuestos sinterizados a $1600{ }^{\circ} \mathrm{C}$, es superior a la correspondiente a cada uno de los dos materiales monolíticos obtenidos a la misma temperatura. Así, la introducción de un $20 \%$ vol. de CNFs a la matriz de $\mathrm{nSiC}$, supone un aumento de casi un $50 \%$ de resistencia a la fractura respecto al material de $\mathrm{nSiC}$ monolítico. Por lo tanto, a pesar de tener unos valores de densidad muy similares las nanofibras de carbono actúan como refuerzo mejorando la resistencia del composite. El material monolítico de $\mathrm{nSiC}$ sinterizado a $1800{ }^{\circ} \mathrm{C}$, presenta una resistencia a la fractura superior a la correspondiente a $1600{ }^{\circ} \mathrm{C}$, y ligeramente inferior a todos los composites. Como se comentó anteriormente, la densificación del carburo de silicio es muy sensible a la temperatura final de sinterización. En resumen, existe una sinergia entre ambos tipos de matrices, pudiendo resumirse en una mejora de la densificación del carburo de silicio por la presencia de las nanofibras de carbono a una temperatura relativamente baja.

En la Figura 4 se muestran las micrografías correspondientes a la superficie de fractura del composite $\mathrm{nSiC}+50 \%$ vol.CNFs, sinterizado a $1600{ }^{\circ} \mathrm{C}$ y a $1800{ }^{\circ} \mathrm{C}$. En ellas se puede apreciar que el tamaño de grano que presenta el nSiC es muy parecido, del orden de pocos nanómetros. A pesar de la similitud en el tamaño de partícula, en ambos materiales, en el caso del composite de $\mathrm{nSiC} / \mathrm{CNF}$ s sinterizado a $1800{ }^{\circ} \mathrm{C}$, se observa una ligera disminución de la porosidad, lo que justifica que aumente su resistencia mecánica. En cualquier caso, la falta de densidad en estos materiales sigue siendo evidente, por lo que se puede aventurar que el margen de mejora en sus prestaciones mecánicas es muy importante, en la medida en que se consiga mejorar su sinterización.

\subsection{Efecto del tiempo de estancia a la máxima temperatura de sinterización: Materiales sinterizados a $1800^{\circ} \mathrm{C}$ con 1 y $30 \mathrm{~min}$ de estancia.}

Con el fin de mejorar la densificación de los composites $\mathrm{nSiC} / \mathrm{CNFs}$, se ha planteado estudiar la influencia que puede tener la modificación en el tiempo de estancia a la máxima temperatura de sinterización $\left(1800^{\circ} \mathrm{C}\right)$. Para ello, se ha llevado a cabo una serie de experimentos en los que se ha incrementado el tiempo de estancia de 1 a 30 minutos. En la Figura 5 se muestran los resultados de la densidad y resistencia a la fractura, tanto del material monolítico de carburo de silicio como de los composites $\mathrm{nSiC} / \mathrm{CNF}$. El error experimental de los valores de la densidad relativa calculados asociados al valor medio fue de $\pm 0.5 \%$ y de la resistencia a la fractura de $\pm 5 \mathrm{MPa}$. A modo de ejemplo, en la Figura 6 se muestra la superficie de fractura del material nSiC-CNFs 50/50\%vol. sinterizado a $1800{ }^{\circ} \mathrm{C}$ durante 30 minutos. En ella, es posible
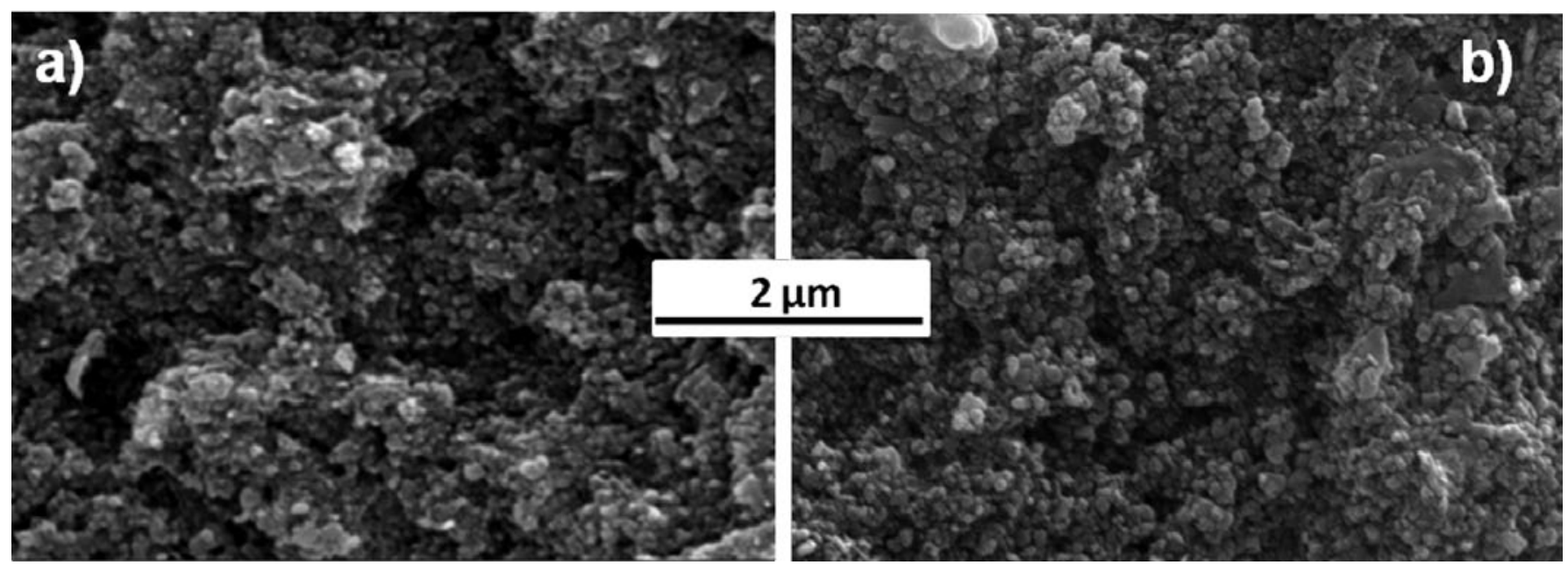

Figura 4. Micrografías MEB correspondientes a la superficie de fractura del composite nSiC $+50 \%$ vol.CNFs, sinterizado mediante SPS a $1600{ }^{\circ} \mathrm{C}$ (a) y $1800^{\circ} \mathrm{C}(\mathrm{b})$. 
distinguir la presencia de nanofibras de carbono en la matriz cerámica.El primer resultado destacable es que se aprecia una mejora en la densidad de los materiales compuestos con altos contenidos en $\mathrm{nSiC}$ (50 y $80 \%$ vol.) mientras que en el caso del material monolítico de $\mathrm{nSiC}$ o el composite con elevados contenidos en nanofibras de carbono, el tiempo de estancia a alta temperatura no parece tener una influencia destacable. Este resultado apoya la idea de que existe una interacción positiva entre ambos tipos de componentes, y que además, a la vista de estos resultados se aprecia que es dependiente del tiempo y de la composición del material. Así, la mejora en la resistencia a la fractura es mucho mayor en el caso de los composites de carburo de silicio reforzado con el menor contenido en nanofibras de carbono (20\%vol.), lo que confirma que, en cierta medida, las CNFs están facilitando la sinterización del carburo de silicio. En este caso, la resistencia mecánica aumenta de 72 a $178 \mathrm{MPa}$ al incrementar el tiempo de estancia de 1 a 30 minutos. Así el hecho de que las nanofibras de carbono jueguen un papel importante en la densificación del carburo de silicio se pone de manifiesto por la falta de mejora en la densidad del material monolítico al aumentar el tiempo de estancia a 30 minutos. Lógicamente, tal y como cabría esperar, no se aprecia una diferencia significativa en las propiedades mecánicas de aquellos materiales en los que la densidad no ha cambiado con el tiempo de estancia. Por lo tanto, de este estudio se puede concluir que, en el sistema $\mathrm{nSiC} / \mathrm{CNFs}$, resulta especialmente interesante la preparación de materiales compuestos con matriz de carburo de silicio reforzados con nanofibras de carbono, ya que éstas facilitan la sinterización del componente cerámico.

\subsection{Propiedades tribológicas}

La preparación superficial de cada composición para el ensayo tribológico se realiza primero con un pulido fino utilizando una muela fina en la máquina Boccadoro ${ }^{\circledR} \mathrm{TM}$ 3-A. Posteriormente, las muestras se pulieron manualmente con diamante hasta obtener rugosidades por debajo de la micra, para ello, se empleó una pulidora de la casa Struers ${ }^{\circledR}$, modelo RotoPol-31. Se comprueba por microscopía óptica que la preparación es uniforme en todas las muestras. Antes de realizar los ensayos se mide la rugosidad mediante el rugosímetro Perthometer M2 de marca Mahr, evaluado según la norma ISO: 4287-1984 con el parámetro de la desviación media aritmética.

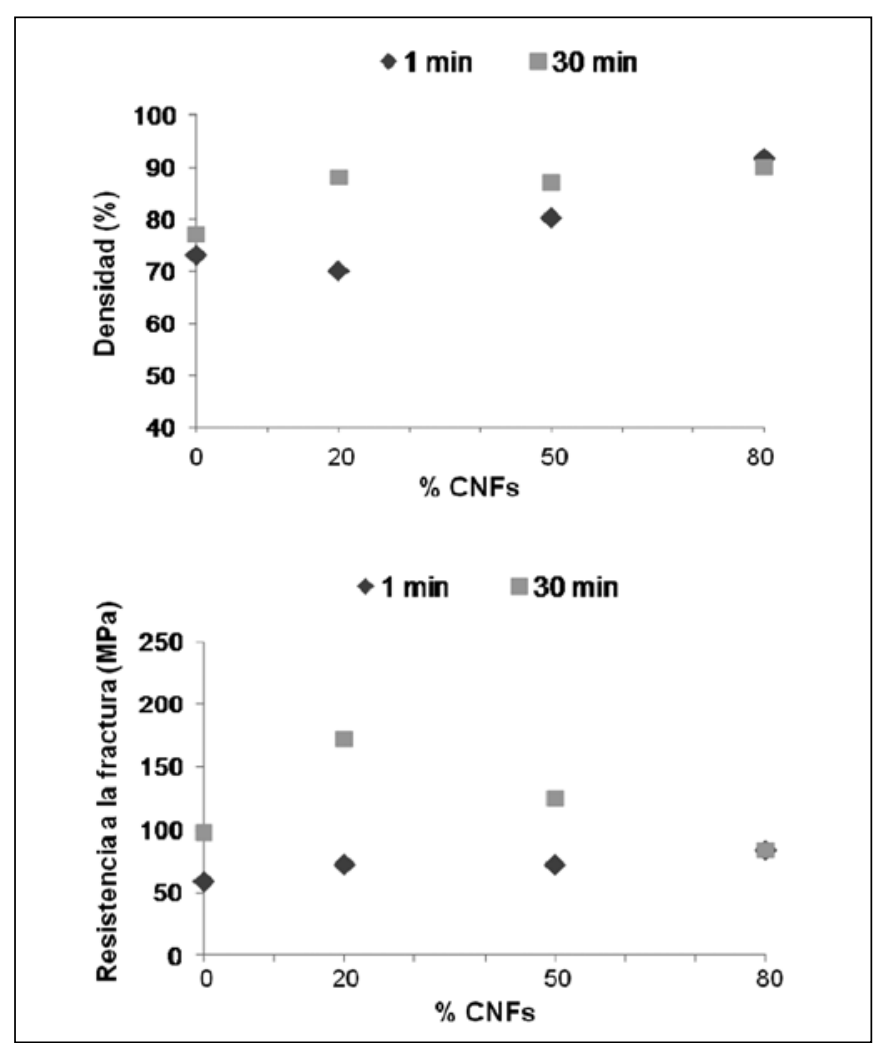

Figura 5. Densidad y resistencia a la fractura del monolítico de nSiC y de los composites $\mathrm{nSiC} / \mathrm{CNFs}$ sinterizados a $1800^{\circ} \mathrm{C}$, con una estancia a la máxima temperatura de 1 y $30 \mathrm{~min}$.

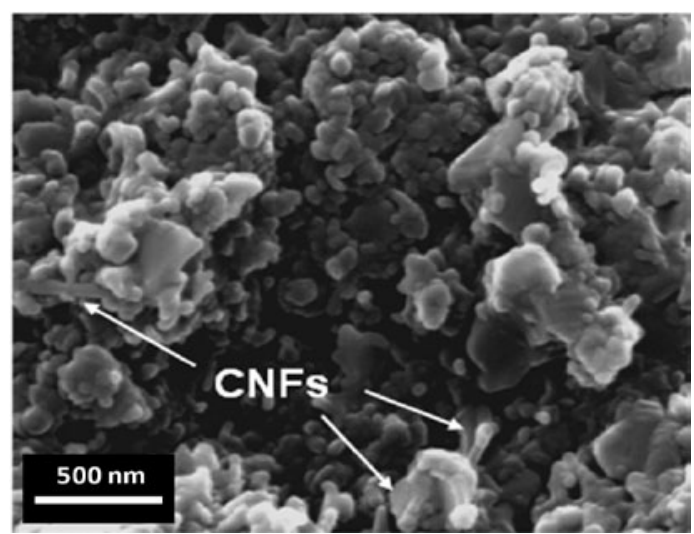

Figura 6. Micrografía de MEB de la superficie de fractura del nanocomposite $\mathrm{nSiC} / \mathrm{CNFs} 50 / 50 \%$.vol sinterizado a $1800{ }^{\circ} \mathrm{C}$ durante 30 minutos.

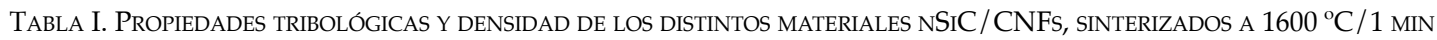

\begin{tabular}{|c|c|c|c|c|c|}
\hline & $100 \%$ vol. nSiC & $\begin{array}{c}80 \% \text { vol. nSiC + } \\
20 \% \text { vol. CNFs }\end{array}$ & $\begin{array}{c}50 \% \text { vol. nSiC + } \\
50 \% \text { vol. CNFs }\end{array}$ & $\begin{array}{c}20 \% \text { vol. nSiC }+ \\
80 \% \text { vol. CNFs }\end{array}$ & $100 \%$ vol. CNFs \\
\hline Coeficiente de fricción & 0.65 & 0.29 & 0.34 & 0.22 & 0.35 \\
\hline Pérdida de volumen $\left(\mathrm{mm}^{3}\right)$ & 2.96 & 1.86 & 0.53 & 0.43 & 0.27 \\
\hline Tasa de desgaste $\left(\mathrm{mm}^{3} / \mathrm{m} \cdot \mathrm{N}\right)$ & $1.97 \cdot 10^{-4}$ & $1.24 \cdot 10^{-4}$ & $3.52 \cdot 10^{-5}$ & $2.86 \cdot 10^{-5}$ & $1.79 \cdot 10^{-5}$ \\
\hline Densidad $(\%)$ & 65.0 & 61.9 & 73.3 & 88.1 & 91.0 \\
\hline
\end{tabular}


Los valores medios del coeficiente de fricción y pérdida de volumen, obtenidos en los diferentes ensayos a los que se han sometido los distintos materiales $\mathrm{nSiC} / \mathrm{CNFs}$, sinterizados a $1600{ }^{\circ} \mathrm{C} / 1 \mathrm{~min}$ se recogen en la Tabla I, donde también se muestra la tasa de desgaste y la densidad relativa de cada material.

Según los datos, el material monolítico de nSiC presenta un coeficiente de fricción muy alto respecto a los otros materiales, ya que se trata de un material cerámico. En cuanto a la tasa de desgaste también es bastante elevada ya que, tanto la resistencia a la fractura como la densidad alcanzada por el mismo son muy bajas y, estos parámetros influyen en el comportamiento al desgaste de los materiales. Por otro lado, se ha observado que la bola de alúmina, durante el ensayo, también experimenta un acusado desgaste, debido probablemente a la generación masiva de debris del material que actúa como un tercer cuerpo. Este factor provoca un aumento del área de contacto real, lo que también contribuye al aumento del coeficiente de fricción.

El composite con un $80 \%$ vol. nSiC presenta un desgaste ligeramente inferior al del monolítico de nSiC. Esta mejora en el comportamiento tribológico, en consonancia con el aumento de resistencia mecánica, resulta indicativo de un mayor grado de sinterización por el efecto de las nanofibras de carbono. En cuanto al valor del coeficiente de fricción, la introducción de un $20 \%$ vol. de nanofibras de carbono hace que este disminuya drásticamente. Este comportamiento se debe a la formación de una película de "debris", la cual está formada por las nanofibras de carbono que actúan como lubricante, disminuyendo de esta forma el coeficiente de fricción. Las nanofibras de carbono debido a este efecto lubricante no solo hacen que disminuya el coeficiente de fricción, sino ayudan a que sea más estable. También cabe destacar en la Tabla I, que a medida que se alcanzan densidades relativas más elevadas y, a su vez valores de resistencia a la fractura superiores, la tasa de desgaste de los materiales disminuye. Se pueden diferenciar, claramente, dos zonas, por una parte los materiales con altos contenidos en nSiC (100 y 80) \% vol. $\mathrm{y}$, por otra parte, los materiales con bajos contenidos en nSiC (50 y 20) \%vol. donde el comportamiento a desgaste viene determinado por las nanofibras de carbono. Por lo tanto, la incorporación de nanofibras de carbono tiene dos efectos positivos simultáneamente, por un lado, mejora de la resistencia al desgaste ya que ayudan a la densificación de los materiales y, por otro lado, reducen el coeficiente de fricción por su acción auto-lubricante. Por lo tanto, es importante alcanzar una densidad lo más cercana a la teórica posible, ya que como se observó anteriormente, este parámetro también influye en la mejora de las propiedades mecánicas.

En la Figura 7, puede observarse la morfología de las superficies de desgaste de los composites de $\mathrm{nSiC} / \mathrm{CNFs}$ con un 80 y un $20 \%$ vol. de $\mathrm{nSiC}$, sinterizados a $1600{ }^{\circ} \mathrm{C} / 1 \mathrm{~min}$, tras los ensayos de fricción.

Los mecanismos de desgaste observados varían significativamente con el contenido en $\mathrm{CNF}_{\text {s, }}$ como resultado de los cambios de densidad, de la resistencia mecánica y, también, del efecto lubricante de las nanofibras. En el composite $80 \%$ vol. nSiC $+20 \%$ vol. CNFs (Figura 7a), el mecanismo de pérdida de material predominante es la fractura intergranular masiva resultado de la falta de cohesión de material por su baja densificación. La superficie de desgaste del composite $20 \%$ vol. nSiC $+80 \%$ vol. CNFs presenta un menor daño, tal y como se observa en la Figura $7 \mathrm{~b}$, donde pueden apreciarse leves signos de microabrasión en la dirección de deslizamiento y fracturas perpendiculares al deslizamiento, que resultan indicativas de un desgaste cohesivo.

En la Tabla 2, se presentan las propiedades tribológicas de los composites nSiC/CNFs (20 y 80) \%vol. CNFs sinterizados a $1800{ }^{\circ} \mathrm{C} / 30 \mathrm{~min}$.

Como se puede observar en dicha tabla el dato más significativo es el de la tasa de desgaste, la cual es inferior a la de los correspondientes materiales sinterizados a $1600^{\circ} \mathrm{C} / 1 \mathrm{~min}$, sobre todo la del composite con un $20 \%$ vol. de CNFs, ya que en este material la densidad ha aumentado considerablemente al igual que la resistencia mecánica cuyo valor es más del doble. En cuanto al coeficiente de fricción, el valor es muy parecido al de los materiales sinterizados a 1600 ${ }^{\circ} \mathrm{C}$, ya que se trata de materiales con la misma composición y ensayados bajo los mismos parámetros.
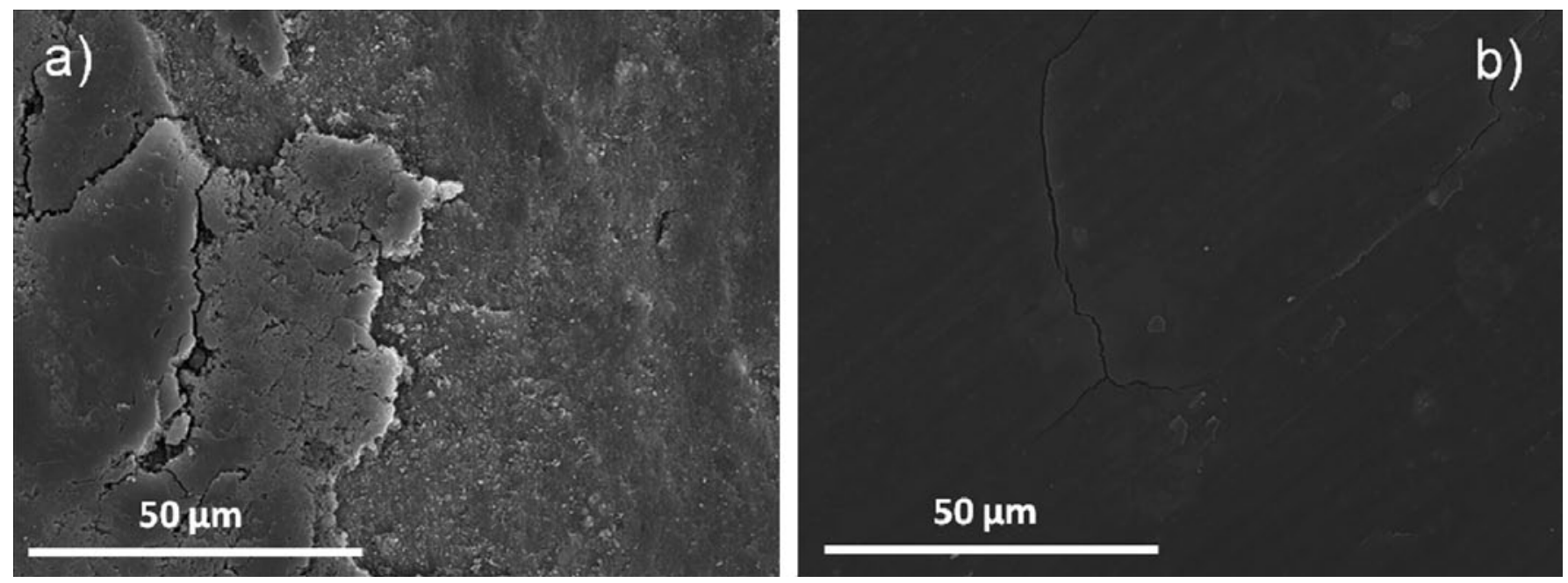

Figura 7. Micrografías de MEB de las superficies desgastadas de los composites nSiC/CNFs: a) $80 / 20$ y b) $20 / 80 \%$ vol. sinterizados a $1600{ }^{\circ} \mathrm{C} / 1$ min. 
TABLA II. PROPIEDADES TRIBOLÓGICAS Y DENSIDAD DE LOS DISTINTOS MATERIALES NSIC/CNFS, SINTERIZADOS A $1800^{\circ} \mathrm{C} / 30 \mathrm{MIN}$.

\begin{tabular}{|c|c|c|}
\hline & $\begin{array}{c}80 \% \text { vol. nSiC + } \\
20 \% \text { vol. CNFs }\end{array}$ & $\begin{array}{c}20 \% \text { vol. nSiC } \\
+ \\
80 \% \text { vol. CNFs }\end{array}$ \\
\hline Coeficiente de fricción & 0.31 & 0.20 \\
\hline Pérdida de volumen $\left(\mathrm{mm}^{3}\right)$ & 0.27 & 0.23 \\
\hline Tasa de desgaste $\left(\mathrm{mm}^{3} / \mathrm{m} \cdot \mathrm{N}\right)$ & $6.80 \cdot 10^{-5}$ & $1.55 \cdot 10^{-5}$ \\
\hline Densidad (\%) & 89.0 & 91.6 \\
\hline
\end{tabular}

La Figura 8 muestra la superficie desgastada de los composites nSiC/CNFs (80/20 y 20/80) \%vol. sinterizados a $1800{ }^{\circ} \mathrm{C} / 30 \mathrm{~min}$. El material con bajo contenido en CNFs presenta un comportamiento frágil típico de los materiales cerámicos (Figura 8a), donde el desgaste se produce por fractura y extracción de grandes fragmentos de material. La mayor consolidación del composite, respecto al sinterizado a $1600{ }^{\circ} \mathrm{C}$, hace que el desgaste sea mucho menor. En el composite $80 \%$ vol. nSiC $+20 \%$ vol. CNFs (Figura 8 b), se observa la formación de una tribocapa de residuos con surcos de microabrasión en la dirección del deslizamiento. A diferencia del material sinterizado a $1600{ }^{\circ} \mathrm{C}$ no se han encontrado evidencias de fractura, lo cual indica que el desgaste está en un estadio previo, resultado de la mayor resistencia al desgaste, en consonancia con los resultados de pérdida de material.

Así pues, una mejora importante a tener en cuenta sería alcanzar una densificación completa de los composites utilizando, por ejemplo, diferentes rutas de síntesis y mezcla de polvos, combinado con la sinterización mediante la técnica de SPS utilizando diferentes parámetros, como es el tiempo de estancia a la máxima temperatura, la temperatura final alcanzada y la rampa de calentamiento/enfriamiento del equipo.

\section{CONCLUSIONES}

El empleo de nanofibras de carbono en la fabricación de composites $\mathrm{nSiC} / \mathrm{CNFs}$, permite obtener materiales con una densidad superior a la de los materiales monolíticos sinterizados en las mismas condiciones. El ajuste de los parámetros de sinterización aumenta el efecto que tienen las nanofibras de carbono sobre la sinterización del carburo de silicio, actuando estas como un "promotor de la sinterización", provocando una notable densificación de los composites con la consiguiente mejora en las propiedades mecánicas. La presencia de las nanofibras de carbono en los composites supone, además, una importante mejora en las propiedades tribológicas de estos materiales compuestos, ya que el coeficiente de fricción se reduce a valores de 0.22-0.35, en la mayoría de los casos. Junto con la disminución en el coeficiente de fricción, se consigue también una importante mejora en la estabilidad del mismo, gracias a la formación de una película de nanofibras de carbono entre la superficie de contacto que actúa como lubricante y esto, unido a la mejora en el procesado aumenta la resistencia al desgaste.

\section{AGRADECIMIENTOS}

Este trabajo ha sido llevado a cabo con la financiación procedente de los proyectos del Plan Nacional MAT200601783 y MAT2007-30989-E y del proyecto del plan regional FICYT PC07-021. También agradecer la ayuda del MICINN para la movilidad de becarios FPI, A. Borrell, en Centros de investigación (Instituto de Tecnología de Materiales) de la Universidad Politécnica de Valencia.

\section{BIBLIOGRAFÍA}

1. E. T. Thostenson, L. Chunyu, T. W. Chou, Nanocomposites in context, Compos. Sci. Technol., 65, 491-516 (2005).

2. K. A. Blakely, Ceramic revolutionary yet arrive via nanothechnology, Am. Ceram. Soc. Bull., 85, 30-32 (2006).

3. M. Di Ventra, S. Evoy, Jr. Heflin, R. James, Introduction to nanoscale science and technology, Ed. Hardcover, (2004).
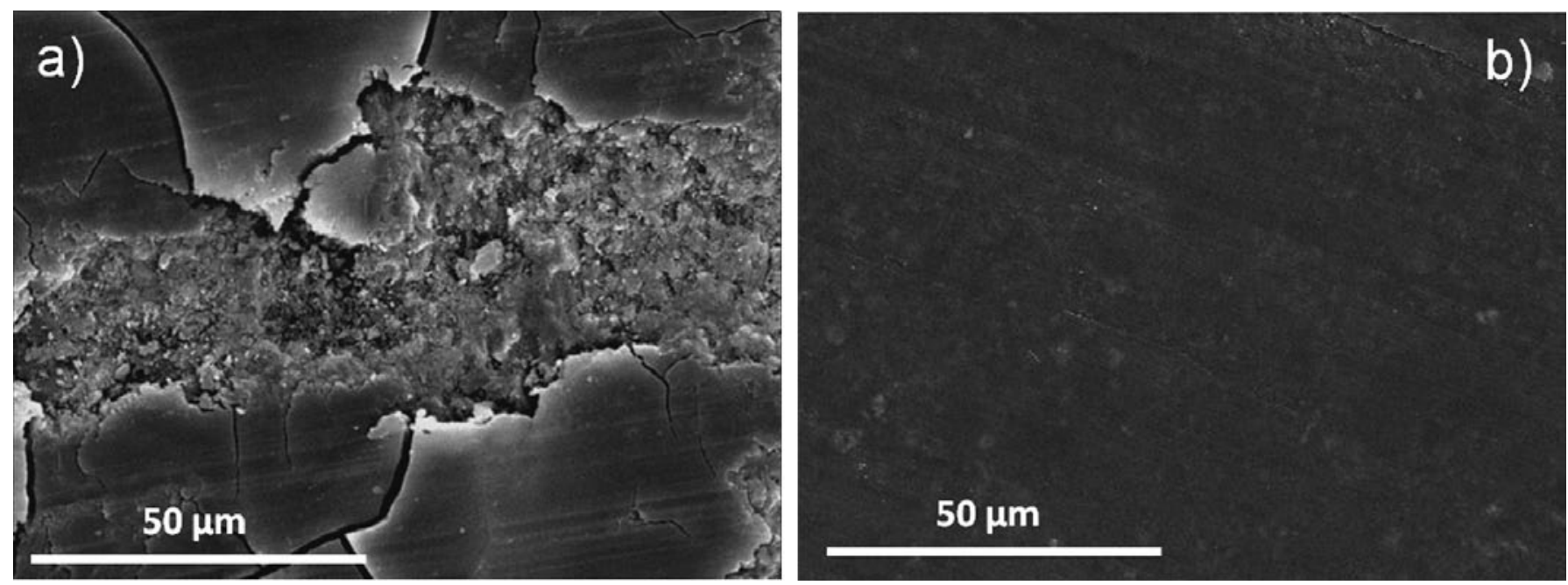

Figura 8. Micrografías de MEB de las superficies desgastadas de los composites nSiC/CNFs: a) $80 / 20$ y b) $20 / 80 \%$ vol. sinterizados a 1800 C 30 min. 
4. I. Kang, Y. Y. Heung, J. H. Kim, J. Won-Lee, R. Gollapudi, S. Subramaniam, S. Narasimhadevara, D. Hurd, G. R. Kirikera, V. Shanov, M. J. Schulz, D. Shi, J. Boerio, S. Mall, M. Ruggles-Wren, Introduction to carbon nanotube and nanofiber smart materials, Compos: Part B., 37, 382-394 (2006).

5. V. Viswanathan, T. Laha, K. Balani, A. Agarwal, S. Seal, Challenges and advances in nanocomposite processing techniques, Mater. Sci. Eng. R., 54, 121-285 (2006).

6. S. K. Lee, Y. C. Kim, C. H. Kim, Microstructural development and mechanical properties of pressureless-sintered $\mathrm{SiC}$ with plate-like grains using $\mathrm{Al}_{2} \mathrm{O}_{3}+\mathrm{Y}_{2} \mathrm{O}_{3}$ additives, J. Mater. Sci., 29, 5321-5326 (1994).

7. E. T. Thostenson, L. Chunyu, Ch. Tsu-Wei, Nanocomposites in context, Compos. Sci. Technol., 65, 491-516 (2005).

8. K. Shimoda, T. Hinoki, A. Kohyama, Effect of carbon nanofibers $(\mathrm{CNFs})$ content on thermal and mechanical properties of $\mathrm{CNFs} / \mathrm{SiC}$ nanocomposites, Compos. Sci. Technol., 70, 387-392 (2010).

9. Ke. Hirota, H. Hara, M. Kato, Mechanical properties of simultaneously synthesized and consolidated carbon nanofiber (CNF)-dispersed $\mathrm{SiC}$ composites by pulsed electric-current pressure sintering, Mater. Sci. Eng., A 458, 216-225 (2007)

10. Y. Wang, Z. Fu, Study of temperature field in spark plasma sintering, Mater. Sci. Eng. B., 90, 34-37 (2002).

11. U. Anselmi-Tamburini, J. E. Garay, Z. A. Munir, Fast low-temperature consolidation of bulk nanometric ceramic materials, Scrip. Mater., 54, 823828 (2006).

12. R. Orrù, R. Licheri, A. M. Locci, A. Cincotti, G. Cao, Consolidation/ synthesis of materials by electric current activated/assisted sintering, Mater. Sci. Eng R. Reports, 63, 4-6 (2009).

13. L. Ci, J. Wei, B. Wei, J. Liang, C. Xu, D. Wu, Carbon nanofibers and single-walled carbon nanotubes prepared by the floating catalyst method, Carbon, 39, 3, 329-335 (2001).
14. A. F. Kirstein, R. M. Woolley, Symmetrical bending of thin circular elastic plates on equally spaced point supports, J. Res. Natl. Bur. Stand, C., 71, 1-10 (1967).

15. F. F. Vitman, V. P. Pukh, A method for determining the strength of sheet glass, Zavod. Lab, 29, 863-867 (1963).

16. ASTM Standard F394-78, STM Annual Book of Standards, Vol. 15.02, Section 16. American Society for Testing and Materials, Philadelphia, PA, 466-490 (1996).

17. A. E. McHale, Phase equilibria diagrams, phase diagrams for ceramists, vol. $\mathrm{X}$, borides, carbides, and nitrides, American Ceramic Society, Columbus, OH, (1994).

18. S. Prochazka, R. M. Scanlan, Effect of boron and carbon on sintering of SiC, J. Am. Ceram. Soc., 58, 72-73 (1975).

19. H. Endo, M. Ueki, H. Kubo, Microstructure and mechanical properties of hot-pressed SiC-TiC composites, J. Mater. Sci., 26, 3769-3774 (1991).

20. E. Ermer, P. Wiesław, S. Ludosław, Influence of sintering activators on structure of silicon carbide, Sol. Stat. Ion., 141-142, 523-528 (2001).

21. A. Borrell, A. Fernández, C. Merino, R. Torrecillas, High density carbon materials obtained at relatively low temperature by spark plasma sintering of carbon nanofibers, Int. J. Mater. Res., 101, 1, 112-118 (2010).

22. A. Borrell, V.G. Rocha, R. Torrecillas, A. Fernández, Surface coating on carbon nanofibers with alumina precursor by different synthesis routes, Compos. Sci. Technol., 71, 18-22 (2011)

23. A. Borrell, V.G. Rocha, R. Torrecillas, A. Fernández, Improvement of carbon nanofibers $/ \mathrm{ZrO}_{2}$ composites properties with a zirconia nanocoating on carbon nanofibers by sol-gel method, J. Am. Ceram. Soc., DOI: 10.1111/j.1551-2916.2010.04354.x

Recibido: 15/11/2010

Aceptado: 1/4/2011 